\title{
Tuning Metamaterials for Applications at DUV Wavelengths
}

\author{
Andrew Estroff and Bruce W. Smith \\ Microsystems Engineering, Rochester Institute of Technology, 77 Lomb Memorial Drive, Rochester, NY 14623, USA \\ Correspondence should be addressed to Andrew Estroff, ace9411@rit.edu
}

Received 9 April 2012; Accepted 23 July 2012

Academic Editor: Xiaoyue Huang

Copyright ( $) 2012$ A. Estroff and B. W. Smith. This is an open access article distributed under the Creative Commons Attribution License, which permits unrestricted use, distribution, and reproduction in any medium, provided the original work is properly cited.

\begin{abstract}
The unique properties of metamaterials, namely, their negative refractive index, permittivity, or permeability, have gained much recent attention. Research into these materials has led to the realization of a host of applications that may be useful to enhance optical nanolithography. A selection of materials has been examined both experimentally and theoretically to verify their support of surface plasmons, or lack thereof, in the DUV spectrum via the attenuated total reflection (ATR) method using the Kretschmann configuration. At DUV wavelengths, materials that were previously useful at mid-UV and longer wavelengths no longer act as metamaterials. Structured materials comprised of alternating layers of aluminum and aluminum oxide $\left(\mathrm{Al}_{2} \mathrm{O}_{3}\right)$, as well as some other absorption-free dielectrics, exhibit metamaterial behavior, as do some elemental materials such as aluminum. These elemental and structured materials exhibit the best properties for use in plasmonic nanolithographic applications. Therefore, a simulator was created to examine material and thickness combinations to generate a tunable metamaterial for use in the DUV. A method for performing plasmonic interference lithography with this metamaterial has been proposed, with calculations showing the potential for half-pitch imaging resolution of $25 \mathrm{~nm}$.
\end{abstract}

\section{Introduction}

A surface plasmon (SP) is an electromagnetic wave carried by free electrons that travels along the boundary between a conductor and a dielectric. Surface plasmons were first noticed experimentally in the early 1900 s by Wood [1] as anomalous drops in the reflected intensity from metalbacked gratings, but it was not until the 1940s that Fano [2] linked this to earlier theory developed by Sommerfeld [3]. In the late 1950s, Ritchie [4] performed experiments on electron energy losses in thin foils, and Pines and Bohm [5-7] discovered that these losses were due to the excitation of conducting electrons, plasma oscillations they named plasmons [8]. In the 1960s, Kretschmann and Raether [9], as well as Otto [10], developed techniques to optically excite surface plasmons via attenuated total reflection (ATR). It was not until the early 1990s that exponential growth in plasmon research began [11].

In 2000, Pendry [12] showed that Veselago's [13] theoretical negative refractive index slab lens was realizable using a negative permittivity material instead, and that this near field imaging system was enabled by surface plasmons.
This discovery encouraged research into plasmonic lithography [14-17].

While the idea of Pendry's perfect lens is of importance and allows deep subwavelength imaging of arbitrary shapes, it requires a complex mask with feature sizes on the same order as those to be imaged. More recently, others have proposed the idea of maskless plasmonic interference lithography, in which two beams are input at opposite sides of a Kretschmann configured prism, creating two counter propagating surface plasmons, resulting in a standing wave $[18,19]$. The pitch for this setup is shown in (1) and is dependent upon the permittivity of the prism, incident angle (resonance angle), and the incident wavelength [20]:

$$
p=\frac{\lambda_{0}}{2 \sqrt{\varepsilon_{d}} \sin \theta}
$$

Much of this research has been performed at mid-UV wavelengths, as silver is a near ideal material for use in that spectrum. However, most currently lithography is performed in the DUV spectrum, and it is our goal to examine 


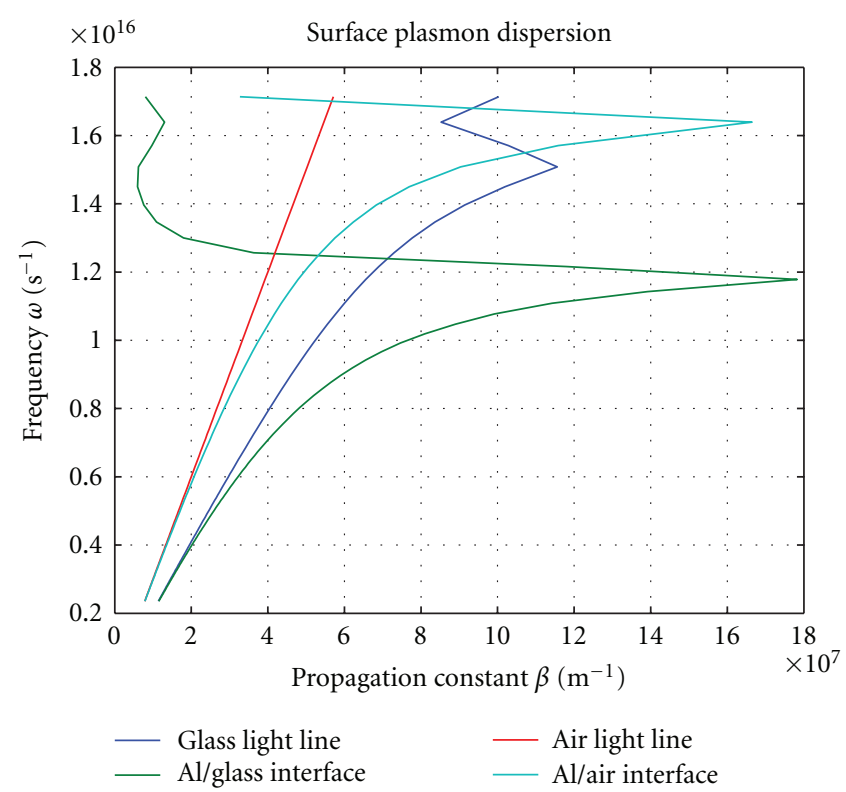

FIgURE 1: Surface plasmon dispersion curves for Kretschmann ATR configuration, calculated using material properties for $\mathrm{Al}$ and $\mathrm{SiO}_{2}$ from RIT material database [22].

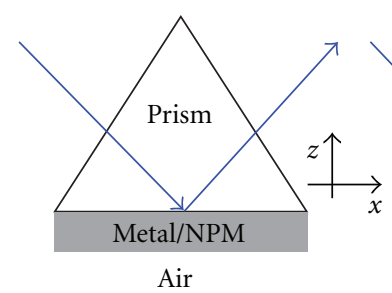

(a)

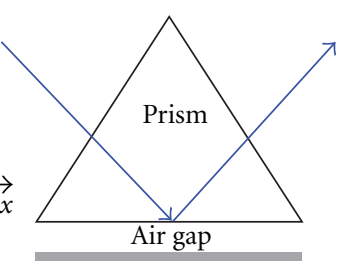

Metal/NPM
Figure 2: Kretschmann configuration (left), Otto configuration (right).

metamaterials that could be used for plasmonic imaging in this regime.

\section{Approach}

The dispersion relation for surface plasmons can be obtained by solving the wave equation and is described well in [21]. The resulting surface plasmon dispersion relation for a single interface is

$$
\beta=\frac{\omega}{c} \sqrt{\frac{\varepsilon_{m} \varepsilon_{d}}{\varepsilon_{m}+\varepsilon_{d}}},
$$

where $\beta$ is the propagation constant, $\omega$ is the wave vector of the incident radiation, and $\varepsilon_{m}$ and $\varepsilon_{d}$ are the dielectric functions of the conductor and dielectric. It should be noted that this dispersion relation is only valid for TM polarization (also known as p polarization) states; surface plasmons are not excited by TE polarization. An example of the dispersion relation for SPs is shown in Figure 1.

Equation (2) shows that the propagation constant becomes asymptotic when the dielectric functions are equal
Layer Maker computes possible MIM stack combinations

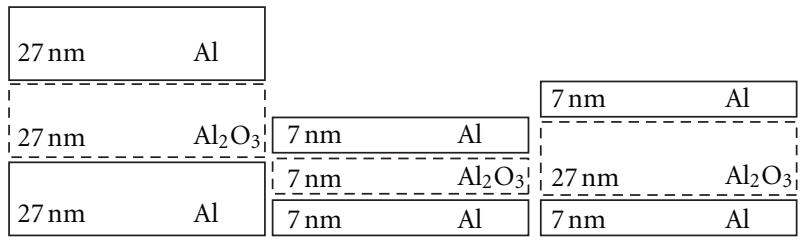

FIGURE 3: Diagram representing Layer Maker application output.

and opposite in sign, for example, $\varepsilon_{m}=-\varepsilon_{d}$. This condition is known as the surface plasmon resonance (SPR). In Figure 1, the red air line represents the dispersion line for radiation in free space, while the SP dispersion curve for the airmetal interface is the light blue curve that lies to the right of it (same convention for glass). At frequencies far below the SPR frequency, the SP propagation constant is relatively small, meaning that the SP wavelength, $\lambda_{\mathrm{sp}}=2 \pi / \operatorname{Re}(\beta)$, is only slightly smaller than the incident radiation. As the frequency of the incident radiation increases to the surface plasmon resonance frequency, the propagation constant increases and approaches infinity at the SPR frequency, meaning that the SP wavelength becomes infinitesimally small. For real metals that are not perfect conductors and exhibit some absorption, the dispersion curves do not become entirely asymptotic, and the dispersion curve sharply moves back to a smaller propagation constant (longer wavelength).

It is apparent from the dispersion curves that a surface plasmon on a simple metal-dielectric interface cannot be excited by light due to the SP propagation constant always being larger than the wave vector of light in the dielectric. However, incident light can be phase matched with the SP by means of prism coupling, a technique referred to as ATR, using either the Kretschmann configuration [9], which has the prism and metal film in direct contact (Figure 2(a)), or the Otto configuration [10], in which a dielectric prism and metal are separated by an air gap (Figure 2(b)). In both configurations, phase matching is accomplished by varying the angle of incidence of the TM-polarized radiation, resulting in a sharp decrease in the reflected intensity at some angle beyond the critical angle. The phase matching occurs when the in-plane wave vector of the reflected beam along the metal surface is the same as the propagation constant; this relationship is shown in (3). The only propagation constants available are those that lie between the air and prism light lines (in Figure 1, this would be between air and glass)

$$
k_{x}=k_{0} n_{p} \sin \theta=\beta .
$$

The surface plasmon dispersion equation shows that a negative permittivity material is needed in conjunction with a dielectric to support surface plasmons. We have previously shown that aluminum is one of the best candidates for use in the DUV spectrum [23]. However, while it is clear that these results demonstrate aluminum supports surface plasmons in the DUV, they need to be considered in conjunction with the plasmon dispersion equation for this ATR setup. Figure 1 shows the dispersion curves for an ATR configuration with an aluminum film and quartz prism. 


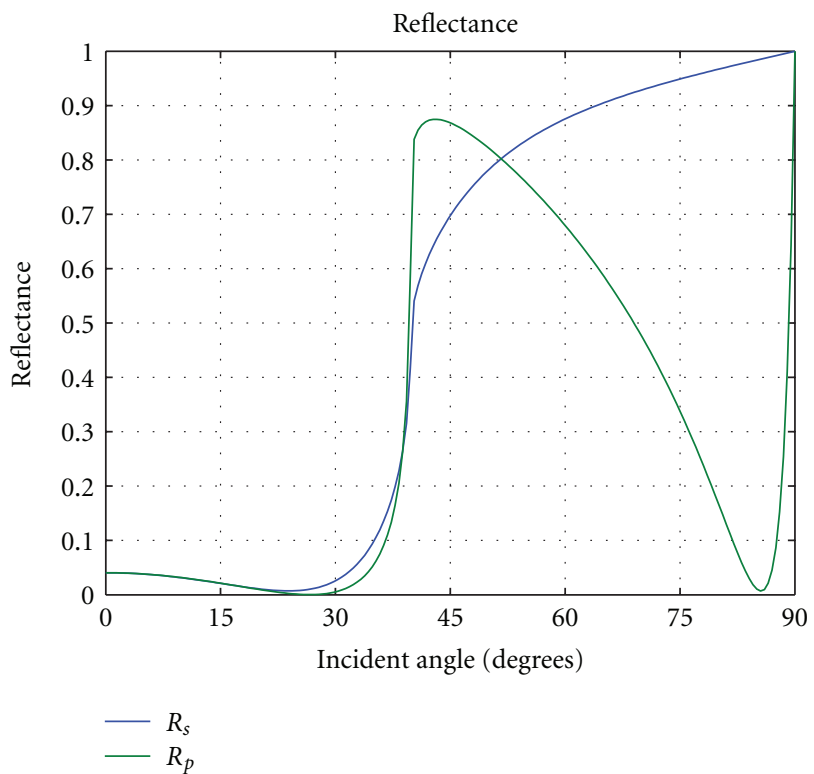

(a)

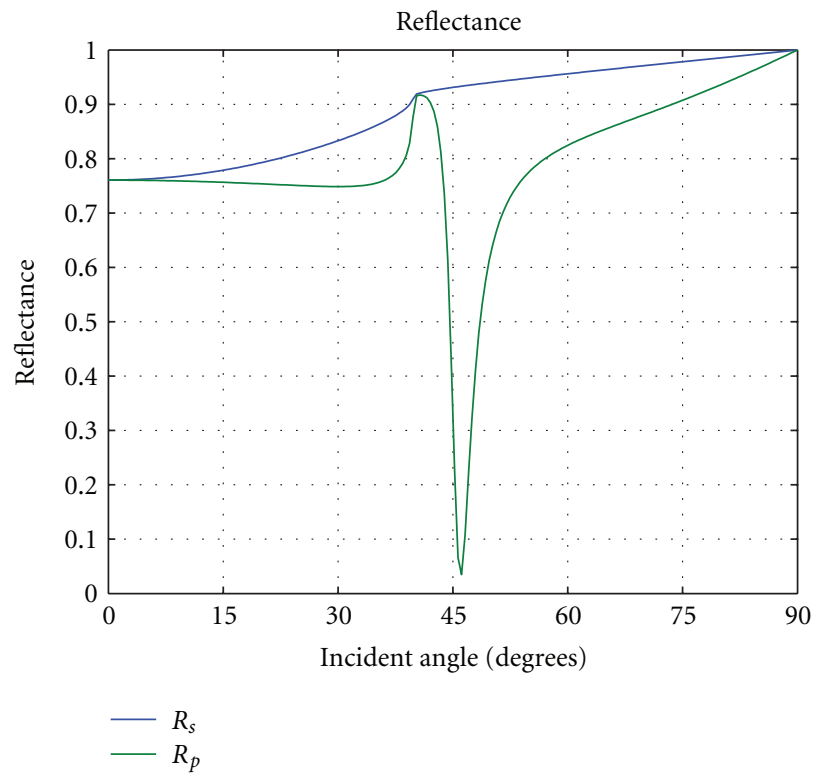

(b)

Figure 4: ATR results for $7 \mathrm{~nm} \mathrm{Al}, 27 \mathrm{~nm} \mathrm{Al}_{2} \mathrm{O}_{3}, 7 \mathrm{~nm} \mathrm{Al}$ stack (left), and single $20 \mathrm{~nm} \mathrm{Al} \mathrm{layer} \mathrm{(right).}$

Stimulator modules Input optical parameters and maximum thicknesses for
materials comprising the film stack into the Layer Maker
application

Import film stack combinations into MATLAB to perform reflection and transmission calculations for each film

Input reflection and transmission data into the Beta Profiler application
MySQL database

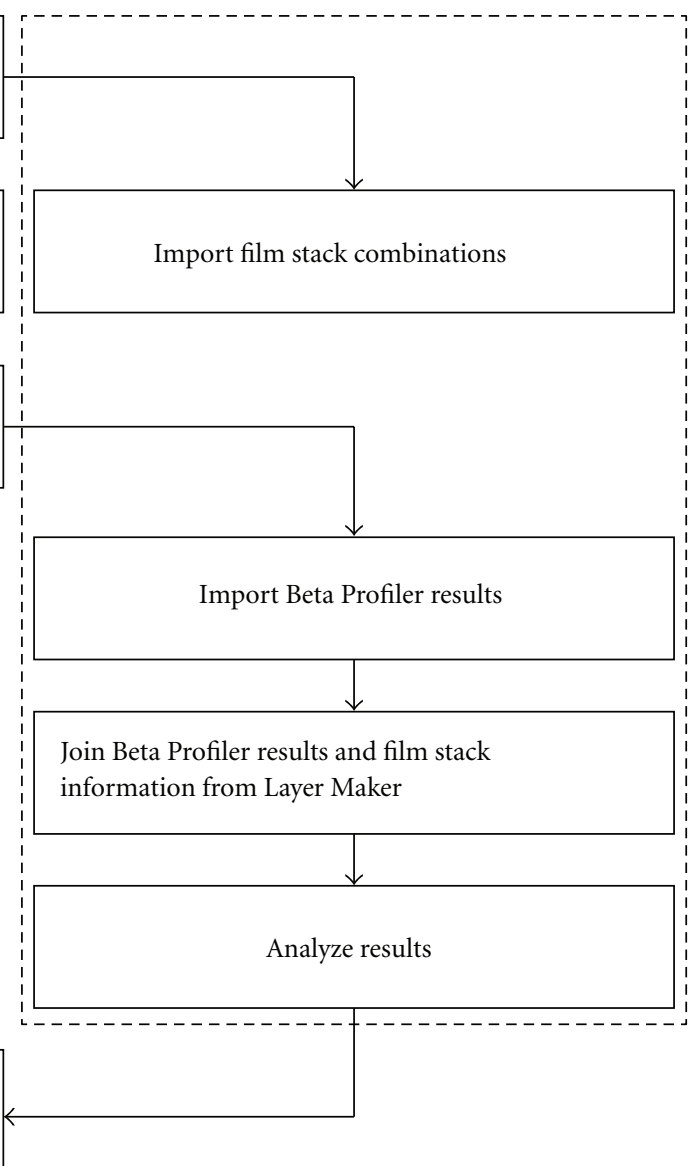

FIgURE 5: Simulator flow chart. 


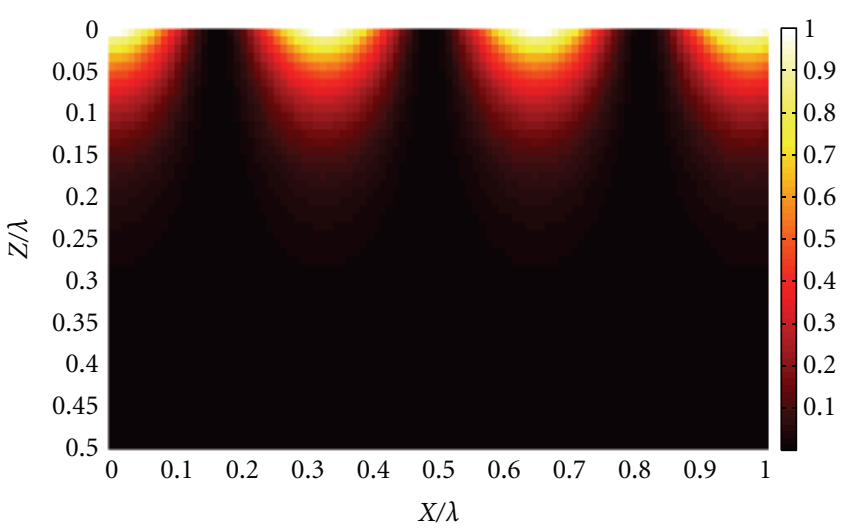

(a)

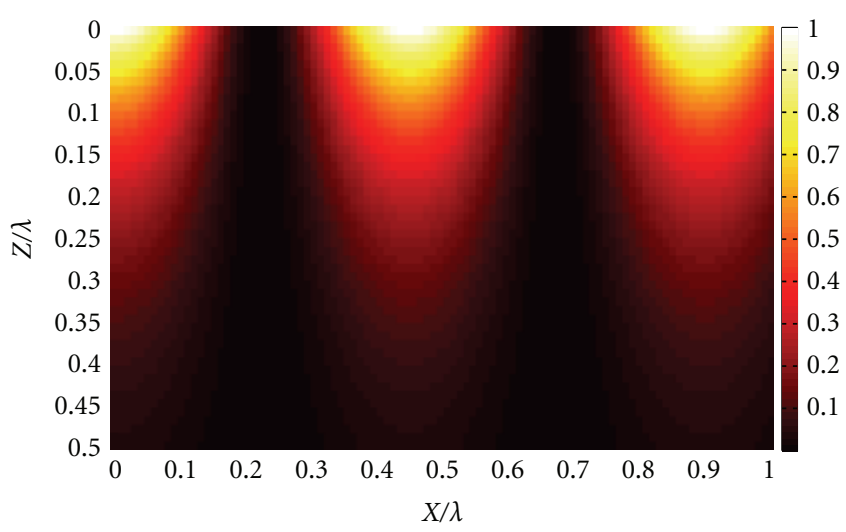

(b)

Figure 6: Electric field intensity profiles below a $7 \mathrm{~nm}-27 \mathrm{~nm}-7 \mathrm{~nm} \mathrm{Al}-\mathrm{Al}_{2} \mathrm{O}_{3}-\mathrm{Al}$ stack (left) and $20 \mathrm{~nm}$ Al film (right) deposited on a quartz prism.

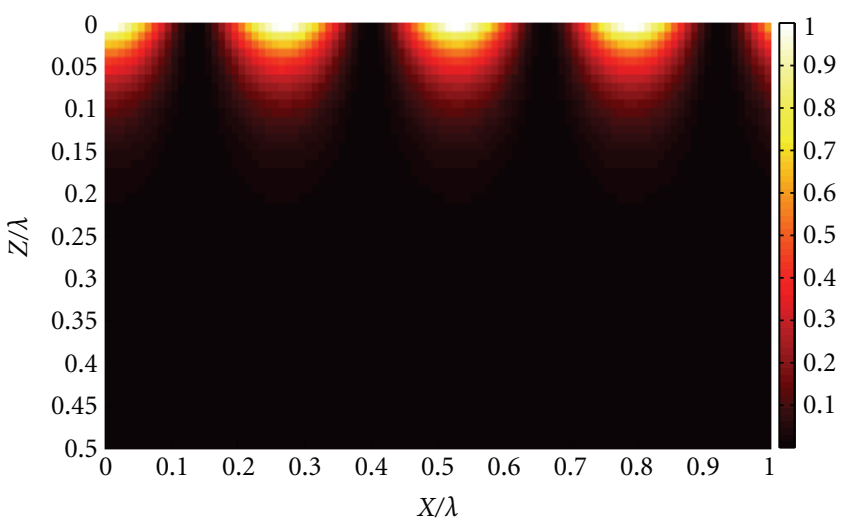

(a)

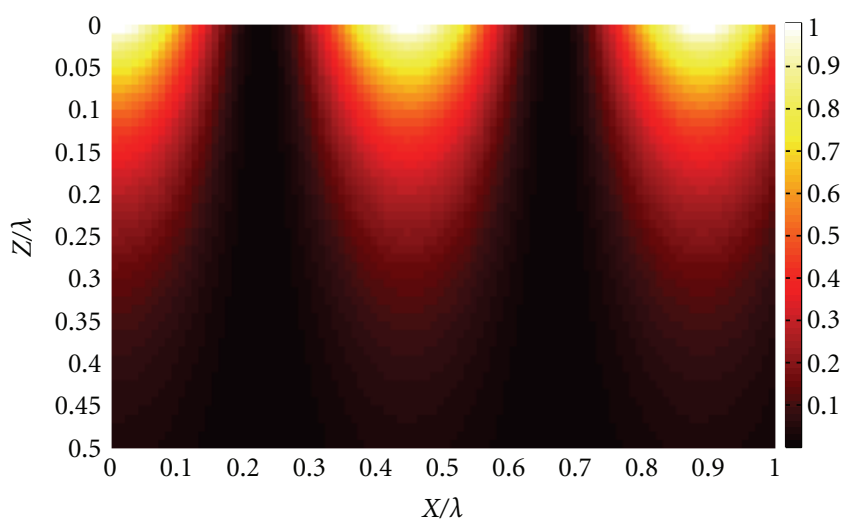

(b)

FIGURE 7: Electric field intensity profiles below $7 \mathrm{~nm}-34 \mathrm{~nm}-7 \mathrm{~nm} \mathrm{Al}-\mathrm{Al}_{2} \mathrm{O}_{3}-\mathrm{Al}$ stack (left) and $20 \mathrm{~nm} \mathrm{Al} \mathrm{film} \mathrm{(right)} \mathrm{deposited} \mathrm{on} \mathrm{a} \mathrm{sapphire}$ prism.

Incident radiation of $193 \mathrm{~nm}$ corresponds to an angular frequency of $\sim 9.7 \times 10^{15} \mathrm{~s}^{-1}$, which means that the accessible surface plasmons are of a wavelength very similar to that of the incident radiation $\left(\lambda_{\mathrm{sp}} \sim 171 \mathrm{~nm}\right)$. Plasmonic interference lithography takes advantage of the fact that at the surface plasmon resonance, the SP wavelength is much smaller than the exciting wavelength. Therefore, a metamaterial needs to be designed to produce a surface plasmon resonance at the desired input frequency.

Pendry has previously demonstrated that it is possible to create a structured material that can be tuned to provide the desired plasma response [24, 25]. Pendry has more recently shown that this structure needs not to be comprised of wires, but that holes in a thin metal film can be designed to provide surface plasmons with arbitrary dispersion [26]. Others have shown that an even simpler design of a layered metalinsulator-metal (MIM) stack can be used to accomplish this [27-31]. Thus, an MIM stack should be examined to provide the desired dispersion characteristics.
Since aluminum is one of the best plasmonic materials in the DUV, and $\mathrm{Al}_{2} \mathrm{O}_{3}$ is a relatively high-index, absorptionfree dielectric, a layered $\mathrm{Al}-\mathrm{Al}_{2} \mathrm{O}_{3}$ system will be examined. A simulator was created to generate and analyze multilayer MIM stacks in order to obtain the largest propagation constants possible, resulting in the shortest plasmon wavelength.

The first module of the simulator is the Layer Maker application, which generates the multilayer stacks to be examined. The refractive index and absorption coefficients of the superstrate (prism), metal, dielectric, and substrate can be input, as well as the maximum total stack thickness to be allowed, the maximum dielectric thickness, and the maximum metal thickness allowed (this is the overall maximum metal thickness, not one individual layer thickness, as it is known in the Kretschmann configuration that if the metal is too thick, SPs cannot couple through it). This application then outputs two files containing all layer combinations within the boundary conditions specified. One file is a MySQL table that will be linked to the output of 
TABLE 1: Joined data in MySQL.

\begin{tabular}{|c|c|c|c|c|c|c|c|c|}
\hline Layer ID & Layers & Metal thick (nm) & Metal count & Dielectric thick (nm) & Dielectric count & Angle (deg) & Reflectance & Beta \\
\hline 15127 & 3 & 5 & 2 & 28 & 1 & 87.51 & 0.0042 & 50738563 \\
\hline 15156 & 3 & 5 & 2 & 29 & 1 & 87.51 & 0.0682 & 50738563 \\
\hline 15098 & 3 & 5 & 2 & 27 & 1 & 87.02 & 0.1021 & 50717534 \\
\hline 15185 & 3 & 5 & 2 & 30 & 1 & 87.02 & 0.2338 & 50717534 \\
\hline 17574 & 3 & 6 & 2 & 27 & 1 & 87.02 & 0.0072 & 50717534 \\
\hline 17551 & 3 & 6 & 2 & 26 & 1 & 86.52 & 0.0358 & 50692685 \\
\hline 17597 & 3 & 6 & 2 & 28 & 1 & 86.52 & 0.1171 & 50692685 \\
\hline 15214 & 3 & 5 & 2 & 31 & 1 & 86.02 & 0.3819 & 50664018 \\
\hline 15069 & 3 & 5 & 2 & 26 & 1 & 85.52 & 0.2362 & 50631535 \\
\hline 17620 & 3 & 6 & 2 & 29 & 1 & 85.52 & 0.2723 & 50631535 \\
\hline 19837 & 3 & 7 & 2 & 26 & 1 & 85.52 & 0.0034 & 50631535 \\
\hline 19856 & 3 & 7 & 2 & 27 & 1 & 85.52 & 0.0898 & 50631535 \\
\hline 15243 & 3 & 5 & 2 & 32 & 1 & 85.03 & 0.4932 & 50595239 \\
\hline 17528 & 3 & 6 & 2 & 25 & 1 & 85.03 & 0.1433 & 50595239 \\
\hline 19818 & 3 & 7 & 2 & 25 & 1 & 85.03 & 0.0348 & 50595239 \\
\hline 17643 & 3 & 6 & 2 & 30 & 1 & 84.53 & 0.4044 & 50555133 \\
\hline 19875 & 3 & 7 & 2 & 28 & 1 & 84.53 & 0.2368 & 50555133 \\
\hline 21971 & 3 & 8 & 2 & 26 & 1 & 84.53 & 0.0294 & 50555133 \\
\hline 15040 & 3 & 5 & 2 & 25 & 1 & 84.03 & 0.3425 & 50511218 \\
\hline 15272 & 3 & 5 & 2 & 33 & 1 & 84.03 & 0.5753 & 50511218 \\
\hline 21954 & 3 & 8 & 2 & 25 & 1 & 84.03 & 0.0040 & 50511218 \\
\hline 21988 & 3 & 8 & 2 & 27 & 1 & 84.03 & 0.1495 & 50511218 \\
\hline 15301 & 3 & 5 & 2 & 34 & 1 & 83.54 & 0.6369 & 50463500 \\
\hline 17666 & 3 & 6 & 2 & 31 & 1 & 83.54 & 0.5052 & 50463500 \\
\hline 19894 & 3 & 7 & 2 & 29 & 1 & 83.54 & 0.3722 & 50463500 \\
\hline 17505 & 3 & 6 & 2 & 24 & 1 & 83.04 & 0.2461 & 50411980 \\
\hline 22005 & 3 & 8 & 2 & 28 & 1 & 83.04 & 0.2939 & 50411980 \\
\hline 23936 & 3 & 9 & 2 & 26 & 1 & 83.04 & 0.0465 & 50411980 \\
\hline 15330 & 3 & 5 & 2 & 35 & 1 & 82.54 & 0.6840 & 50356665 \\
\hline 17689 & 3 & 6 & 2 & 32 & 1 & 82.54 & 0.5813 & 50356665 \\
\hline
\end{tabular}

the other applications via an identity string assigned to each MIM stack. The second file contains a machine readable instruction file for the second application. An example of this table is shown in Figure 3. It should be noted that this application generates stacks comprised of many layers, not simply a 3-layer MIM stack. We are only examining a 3-layer stack here as it is most likely the easiest to fabricate.

The second application is the Matrix Material Calculator (MMC) and is written in MATLAB. It reads the instruction file from the Layer Maker application and calculates reflection profiles through angle for each generated stack. These calculations are performed using matrix methods described by Macleod [32], shown in (4) through (10). The substrate is air, and the initial medium is the quartz prism. The characteristic admittance of each layer is represented as $y_{j}$ and consists of the optical parameters of the layer. The oblique optical admittance is represented by $\eta$ and must be calculated for each polarization state. The film phase factor is $\delta$. The amplitude reflection coefficient $\rho$ can be calculated from the characteristic matrix of the film stack (4), and from this result, the reflectance can be obtained (shown in (10)). With oblique incidence and absorbing materials, care must be taken to ensure that the solutions for $\eta$ for TE polarization states and $\delta$ are in the fourth quadrant to obtain the correct solution (positive real, negative imaginary):

$$
\begin{gathered}
{\left[\begin{array}{l}
B \\
C
\end{array}\right]=\left\{\prod_{j=1}^{q}\left[\begin{array}{cc}
\cos \delta_{j} & \frac{i \sin \delta_{j}}{\eta_{j}} \\
i \eta_{j} \sin \delta_{j} & \cos \delta_{j}
\end{array}\right]\right\}\left[\begin{array}{c}
1 \\
\eta_{\mathrm{sub}}
\end{array}\right],} \\
y_{j}=n-i k \\
\eta_{j_{s}}=\sqrt{n_{j}^{2}-k_{j}^{2}-n_{0}^{2} \sin ^{2} \theta_{0}-2 i n_{j} k_{j}}, \\
n_{j_{p}}=\frac{y_{j}^{2}}{n_{j_{s}}} \\
\delta_{j}=\frac{2 \pi d}{\lambda} \sqrt{n_{j}^{2}-k_{j}^{2}-n_{0}^{2} \sin ^{2} \theta_{0}-2 i n_{j} k_{j}}, \\
\rho=\frac{y_{0} B-C}{y_{0} B+C} \\
R=\rho \rho^{*}
\end{gathered}
$$

The third application is the Beta Profiler. It analyzes the reflection profiles and calculates the angle at which the 
reflection minimum occurs, the reflectance value, and the value of the propagation constant (from (3)). This data is also stored in a MySQL table. By itself, this data is not inherently useful. However, using join statements in MySQL, this data can be merged with the table from the Layer Maker application and sorted to display results that are useful.

Since outputs of the MMC and the Beta Profiler are stored in a MySQL databases it is possible to quickly sort though the data to find optimal cases and analyze the results. An example of a joined output from the Layer Maker and Beta Profiler is shown in Table 1.

The fourth application is the RT Profiler, which is uses the Layer ID to test cases that appear promising. The results shown in Figure 4 are the output of the RT Profiler application. They show that a 3-layer stack comprised of $7 \mathrm{~nm}-27 \mathrm{~nm}-7 \mathrm{~nm}$ of $\mathrm{Al}-\mathrm{Al}_{2} \mathrm{O}_{3}$ - $\mathrm{Al}$ yields a metamaterial with a shorter plasmon wavelength than is capable with just aluminum ( $124 \mathrm{~nm}$ compared to $171 \mathrm{~nm}$ ). A summary of the simulator is shown in the flow chart in Figure 5.

Since the final layer of the tunable metamaterial proposed is $\mathrm{Al}$, it is understood that it will oxidize. The Layer Maker application has been modified to allow an asymmetric stack to be simulated. This makes it possible to simulate our metamaterial stack with a surface oxide layer present, or with a capping material comprised of some other element that may offer more desirable optical properties than an aluminum oxide.

There are several benefits of this simulation approach. Firstly, this matrix method of calculation provides fast results. Simulations of 1 single-layer ATR stack in FDTD software took on the order of a minute to complete. Using this matrix method-based simulator, 50,000 different thin film stack combinations were completed in approximately 90-120 minutes. Secondly, it is quite flexible. Any optical constants desired can be input, and it has been modified further to allow for an asymmetric stack to be input. It has also been modified to allow the optical parameters and thicknesses to vary in order to help model errors that will be seen during fabrication. The joined output data allows one to see the fabrication tolerances allowed to achieve an acceptable result.

The thin film matrix calculations used throughout this document can also be used to calculate the electric field intensity below the stack, which would be representative of imaging possible for plasmonic interference nanolithography. Results for the configurations in Figure 4 are shown in Figure 6. The resulting pitches of $62 \mathrm{~nm}$ and $84 \mathrm{~nm}$ are similar to those calculated using (1).

If a higher index prism is used, an even smaller plasmon wavelength can be achieved. Figure 7 shows the electric field intensity for a $7 \mathrm{~nm}-34 \mathrm{~nm}-7 \mathrm{~nm} \mathrm{Al}-\mathrm{Al}_{2} \mathrm{O}_{3}-\mathrm{Al}$ stack using a sapphire prism $(n=1.92)$, resulting in a $50 \mathrm{~nm}$ pitch (the ATR profile is nearly identical to that for the quartz prism example shown in Figure 4). The resulting pitch for the $20 \mathrm{~nm}$ Al film with the sapphire prism is still nearly identical to that with the quartz prism, due to the fact that the higher index of the prism is offset by a $10^{\circ}$ shift, from $\sim 46^{\circ}$ down to $\sim 36^{\circ}$, in the resonance angle.

\section{Conclusion}

Little research in metamaterials and plasmonics has been done in the DUV spectrum. This paper demonstrates a simulator developed using thin film matrix methods to create tunable metamaterials for the DUV spectrum. It showed that a quartz prism coated with a $7 \mathrm{~nm}-27 \mathrm{~nm}$ $7 \mathrm{~nm} \mathrm{Al}-\mathrm{Al}_{2} \mathrm{O}_{3}-\mathrm{Al}$ thin film stack or a sapphire prism coated with $7 \mathrm{~nm}-34 \mathrm{~nm}-7 \mathrm{~nm} \mathrm{Al}-\mathrm{Al}_{2} \mathrm{O}_{3}-\mathrm{Al}$ is possible solutions, providing more desirable propagation constants, and thus shorter plasmon wavelengths for use in DUV plasmonic interferometric imaging.

\section{References}

[1] R. W. Wood, "On a remarkable case of uneven distribution of light in a diffraction grating spectrum," Philisophical Magazine, vol. 4, pp. 396-402, 1902.

[2] U. Fano, "The theory of anomalous diffraction gratings and of quasi-stationary waves on metallic surfaces (sommerfeld's waves)," Journal of the Optical Society of America, vol. 31, no. 3, pp. 213-222, 1941.

[3] A. Sommerfeld, "Ueber die fortpflanzung elektrodynamischer wellen längs eines drahtes," Annalen der Physik, vol. 303, no. 2, pp. 233-290, 1899.

[4] R. H. Ritchie, "Plasma losses by fast electrons in thin films," Physical Review, vol. 106, no. 5, pp. 874-881, 1957.

[5] D. Bohm and D. Pines, "A collective description of electron interactions. i. magnetic interactions," Physical Review, vol. 82, no. 5, pp. 625-634, 1951.

[6] D. Pines and D. Bohm, "A collective description of electron interactions: ii. Collective vs individual particle aspects of the interactions," Physical Review, vol. 85, no. 2, pp. 338-353, 1952.

[7] D. Bohm and D. Pines, "A collective description of electron interactions: III. Coulomb interactions in a degenerate electron gas," Physical Review, vol. 92, no. 3, pp. 609-625, 1953.

[8] C. T. Walker, "Who named the -oN's?" American Journal of Physics, vol. 38, p. 1380, 1970.

[9] E. Kretschmann and H. Raether, "Radiative decay of non radiative surface plasmons excited by light," Zeitschrift Fur Naturforschung, vol. 23A, pp. 2135-2136, 1968.

[10] A. Otto, "Excitation of nonradiative surface plasma waves in silver by the method of frustrated total reflection," Zeitschrift für Physik, vol. 216, no. 4, pp. 398-410, 1968.

[11] M. L. Brongersma and P. G. Kik, Surface Plasmon Nanophotonics, vol. 131, Springer, 2007.

[12] J. B. Pendry, "Negative refraction makes a perfect lens," Physical Review Letters, vol. 85, no. 18, pp. 3966-3969, 2000.

[13] V. G. Veselago, "The electrodynamics of substances with simultaneously negative values of epsilon and mu," Soviet Physics Uspekhi, vol. 10, no. 4, pp. 509-514, 1968.

[14] N. Fang, H. Lee, C. Sun, and X. Zhang, "Sub-diffractionlimited optical imaging with a silver superlens," Science, vol. 308, no. 5721, pp. 534-537, 2005.

[15] R. J. Blaikie and D. O. S. Melville, "Imaging through planar silver lenses in the optical near field," Journal of Optics A, vol. 7, no. 2, pp. S176-S183, 2005.

[16] D. O. S. Melville and R. J. Blaikie, "Super-resolution imaging through a planar silver layer," Optics Express, vol. 13, no. 6, pp. 2127-2134, 2005. 
[17] D. O. S. Melville and R. J. Blaikie, "Analysis and optimization of multilayer silver superlenses for near-field optical lithography," Physica B, vol. 394, no. 2, pp. 197-202, 2007.

[18] X. Guo and Q. Dong, "Coupled surface plasmon interference lithography based on a metal-bounded dielectric structure," Journal of Applied Physics, vol. 108, no. 11, Article ID 113108, 2010.

[19] K. V. Sreekanth and V. M. Murukeshan, "Large-area maskless surface plasmon interference for one- and two-dimensional periodic nanoscale feature patterning," Journal of the Optical Society of America A, vol. 27, no. 1, pp. 95-99, 2010.

[20] A. Passian, A. Wig, A. L. Lereu et al., "Probing large area surface plasmon interference in thin metal films using photon scanning tunneling microscopy," Ultramicroscopy, vol. 100, no. 3-4, pp. 429-436, 2004.

[21] S. A. Maier, Plasmonics: Fundamentals and Applications, Springer, 2007.

[22] "RIT Nanolithograpy Research Labs > Optical Properties of Thin Films," http://www.rit.edu/kgcoe/microsystems/lithography/thinfilms/thinfilms/thinfilms.html.

[23] A. Estroff, N. V. Lafferty, P. Xie, B. W. Smith et al., "Metamaterials for enhancement of DUV lithography," in Optical Microlithography XXIII, vol. 7640 of Proceedings of the SPIE, pp. 76402W-76402W-10, 2010.

[24] J. B. Pendry, A. J. Holden, D. J. Robbins, and W. J. Stewart, "Low frequency plasmons in thin-wire structures," Journal of Physics Condensed Matter, vol. 10, no. 22, pp. 4785-4809, 1998.

[25] J. B. Pendry, A. J. Holden, W. J. Stewart, and I. Youngs, "Extremely low frequency plasmons in metallic mesostructures," Physical Review Letters, vol. 76, no. 25, pp. 4773-4776, 1996.

[26] J. B. Pendry, L. Martín-Moreno, and F. J. Garcia-Vidal, "Mimicking surface plasmons with structured surfaces," Science, vol. 305, no. 5685, pp. 847-848, 2004.

[27] E. N. Economou, "Surface plasmons in thin films," Physical Review, vol. 182, no. 2, pp. 539-554, 1969.

[28] K. R. Welford and J. R. Sambles, "Coupled surface plasmons in a symmetric system," Journal of Modern Optics, vol. 35, no. 9, pp. 1467-1483, 1988.

[29] G. J. Kovacs and G. D. Scott, "Optical excitation of surface plasma waves in layered media," Physical Review B, vol. 16, no. 4, pp. 1297-1311, 1977.

[30] G. J. Kovacs and G. D. Scott, "Attenuated total reflection angular spectra of a system of alternating plasma-dielectric layers," Applied Optics, vol. 17, no. 22, pp. 3627-3635, 1978.

[31] R. Yang, X. Huang, and Z. Lu, "Arbitrary super surface modes bounded by multilayered metametal," Micromachines, vol. 3, no. 1, pp. 45-54, 2012.

[32] H. A. Macleod, Thin-Film Optical Filters, CRC Press, 2001. 

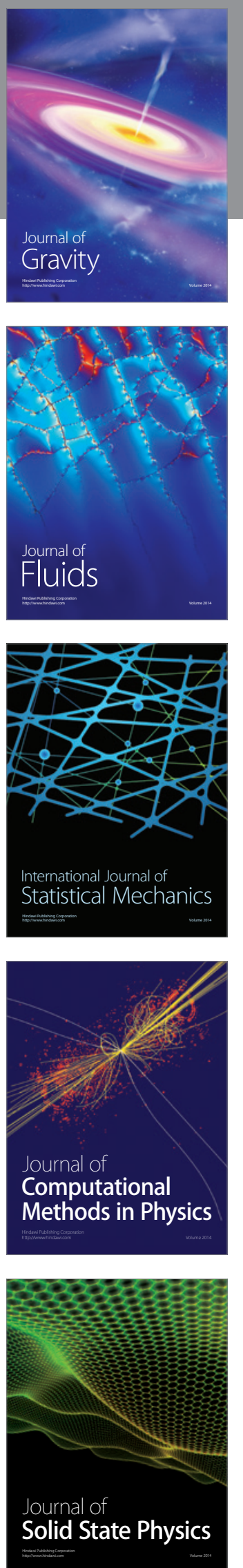

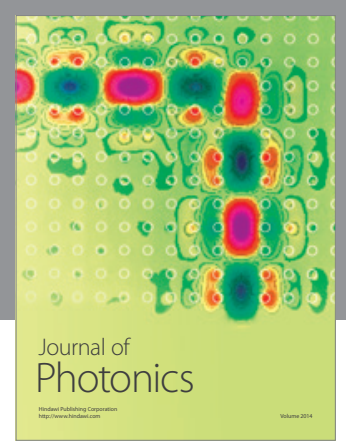

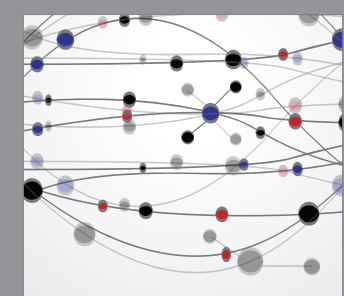

The Scientific World Journal
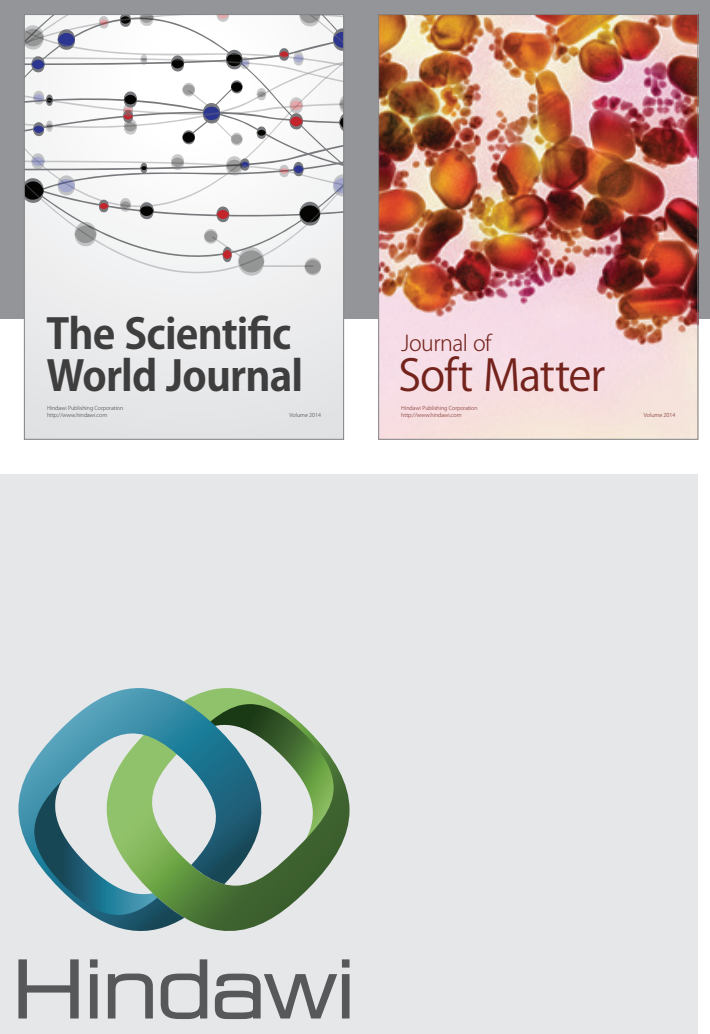

Submit your manuscripts at

http://www.hindawi.com
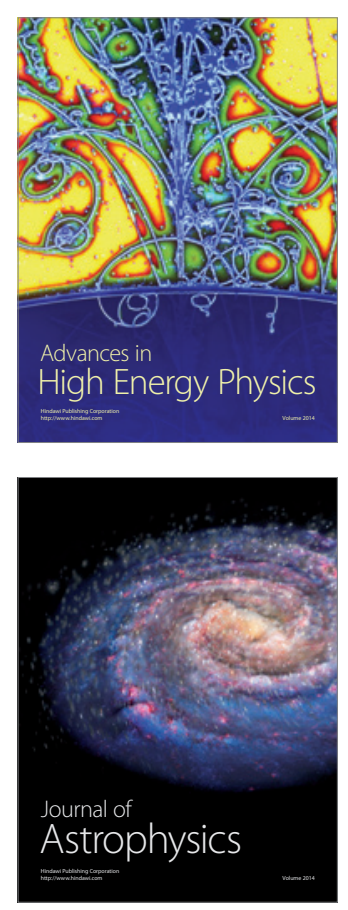
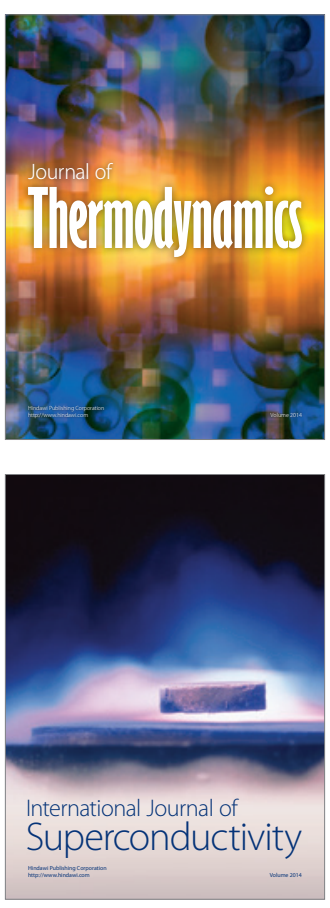
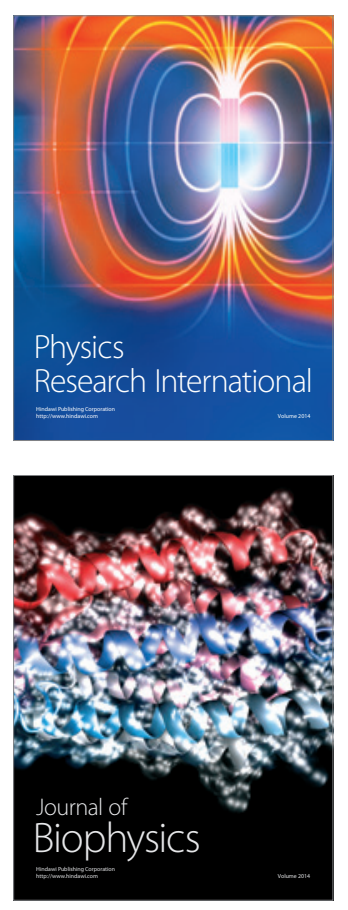
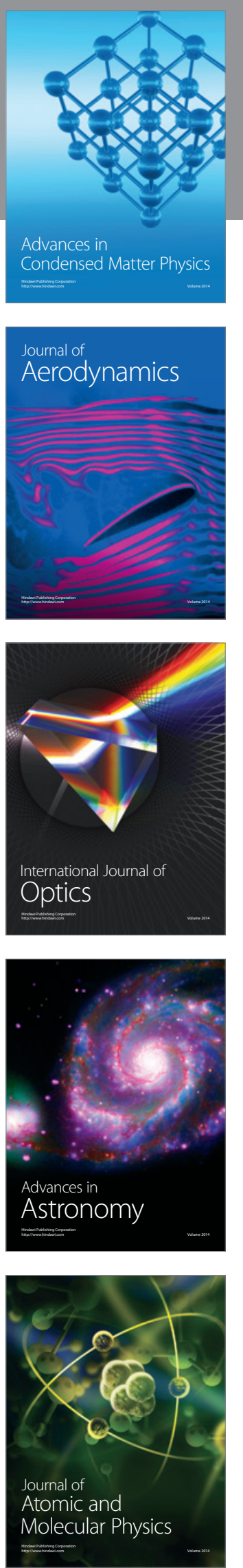